\title{
Repertoire of mouse ectodysplasin-A (EDA-A) isoforms
}

\author{
Tsuyoshi Hashimoto, Chang-Yi Cui, David Schlessinger* \\ Laboratory of Genetics, NIH/National Institute on Aging, Baltimore, MD 21224, USA
}

Received 22 August 2005; received in revised form 8 November 2005; accepted 8 November 2005

Available online 18 January 2006

Received by V. Larionov

\begin{abstract}
Mutations in ectodysplasin-A (EDA) cause loss of hair, sweat glands, and teeth in man and mouse. Isoform EDA-A1 protein shows partial rescue of the affected Tabby mouse phenotypes, suggesting that other isoforms may be required for full function. We describe genomic structure for five EDA isoforms, EDA-A1', A5, A5', A6, and A6', in addition to the previously known EDA-A1, A2, A3, and A4. The novel isoforms together account for $\sim 12 \%$ of total EDA transcripts. The most different, EDA-A6 and A6', which lack the critical domain for interaction with NF$\kappa \mathrm{B}$-activating receptors, were nevertheless confirmed to be present in mouse and human skin tissue. Other isoforms, EDA-A5 and A5', for example, activated NF- $\mathrm{B}$ through receptors EDAR and XEDAR. These properties make new isoforms candidates for modulators of EDA function.
\end{abstract}

Published by Elsevier B.V.

Keywords: Alternative splicing; Ectodermal dysplasia; EDAR; NF-кB; TROY; XEDAR

\section{Introduction}

The EDA gene is mutated in Tabby mouse and in human Xlinked anhidrotic ectodermal dysplasia (OMIM305100), resulting in rudimentary teeth, absence of sweat glands and hair (Kere et al., 1996; Ferguson et al., 1997; Srivastava et al., 1997). Several isoforms generated by alternative splicing are all type II membrane proteins with collagenous and TNF domains in their extracellular portion, giving rise to TNF superfamily ligands by cleavage at a furin protease site (Ezer et al., 1999; Mikkola et

Abbreviations: bp, base pair(s); cDNA, DNA complementary to RNA; EDA, ectodysplasin-A or anhidrotic ectodermal dysplasia; EDAR, ectodysplasin-A receptor; HA, hemagglutinin; IP, immunoprecipitation; kb, kilobase pair(s); LINGO-1, LRR and Ig domain-containing, Nogo Receptor-interacting protein; LRRN6A, leucine rich repeat neuronal 6A; Luc, luciferase; NF- $\kappa \mathrm{B}$, nuclear factor $\kappa \mathrm{B}$; $\mathrm{NgR}$, nogo-66 receptor; nt, nucleotide(s); ORF, open reading frame; PCR, polymerase chain reaction; RTN4R, reticulon 4 receptor; RT-PCR, polymerase chain reaction subsequent to reverse transcroption; TAJ, toxicity and c-Jun N-terminal kinase inducer; TNF, tumor necrosis factor; UTR, untranslated region(s); TRADE, tumor necrosis factor receptor family member associated with death signaling; TROY, tumor necrosis factor receptor superfamily expressed on the mouse embryo; WB, Western blot; XEDAR, X-linked ectodysplasin-A2 receptor.

* Corresponding author. Tel.: +1 410558 8331; fax: +1 4105588331.

E-mail address: schlessingerd@grc.nia.nih.gov (D. Schlessinger). al., 1999; Chen et al., 2001; Elomaa et al., 2001; Schneider et al., 2001). In addition to the two longest "EDA-A" isoforms, EDA-A1 and-A2 in human, two additional isoforms, A3 and A4, have been reported in mouse (Srivastava et al., 1997; Bayes et al., 1998; Mikkola et al., 1999). EDA-A1 and A2 isoforms interact with cognate receptors EDAR and XEDAR, respectively, leading to the activation of the NF-kB pathway (Yan et al., 2000; Kumar et al., 2001; Koppinen et al., 2001; Elomaa et al., 2001; Schneider et al., 2001; Gaide and Schneider, 2003; Shindo and Chaudhary, 2004; Chang and Chaudhary, 2004). However, receptors for other isoforms have not been determined, and the full range of isoforms has remained unknown.

Other isoforms have become more intriguing because an EDA-A1 transgene or EDA-A1 recombinant protein restore some skin appendages including guard hair, tail hair, ear hair, and sweat glands to Tabby mice; but meibomian glands and tooth abnormalities were only partially restored, and other skin appendages, such as zigzag hair and preputial glands, were not restored (Srivastava et al., 2001; Cui et al., 2003; Gaide and Schneider, 2003). Furthermore, an EDA-A1 transgene in wildtype mice produced some aberrant skin and tooth phenotypes (Mustonen et al., 2003; Cui et al., 2003, 2005; Zhang et al., 2003; Newton et al., 2004). These observations indicate that the EDA-A1/EDAR pathway is necessary but not sufficient for 
complete EDA function, and that other isoforms may supplement the action of EDA-A1.

The other major known isoform, EDA-A2, is unlikely to be positively involved: a transgene or EDA-A2 recombinant protein failed to rescue any of the Tabby phenotypes (Cui et al., 2003; Gaide and Schneider, 2003); and although mutation of EDAR or its downstream proteins produced Tabby phenotypes in human and mouse, an XEDAR knockout did not (Headon and Overbeek, 1999; Monreal et al., 1999; Yan et al., 2002; Naito et al., 2002; Courtois et al., 2003; Ohazama et al., 2004; Newton et al., 2004).

To extend the catalogue of other potentially involved EDA-A isoforms, we cloned and analyzed EDA-A cDNAs from mouse keratinocytes. Five new alternative splice variants were found. We demonstrated that several of them bound to TNF superfamily receptors and activated the EDA targeted NF- $\kappa \mathrm{B}$ pathway. However, the isoforms showed modification or truncation of the critical TNF domain, suggesting that they could modify interaction of TNF ligands with TNF superfamily receptors, acting cooperatively or competitively in EDA function.

\section{Materials and methods}

\subsection{Cell culture}

Mouse skin keratinocytes derived from C57BL/6 mice (Cellntec Advanced Cell Systems, Bern, Switzerland) were used for cloning EDA-A isoforms. The cells were maintained in CnT-02 medium (Cellntec Advanced Cell Systems) supplemented with $100 \mathrm{U} / \mathrm{ml}$ of penicillin, $100 \mu \mathrm{g} / \mathrm{ml}$ of streptomycin, and $0.25 \mu \mathrm{g} / \mathrm{ml}$ of amphotericin B (Invitrogen, Carlsbad, CA) in a $\mathrm{CO}_{2}$ incubator at $35^{\circ} \mathrm{C}$. HEK 293 T cells (ATCC; CRL-11268) were used for luciferase reporter assays and immunoprecipitation. Cells were maintained in Dulbecco's modified Eagle medium supplemented with $10 \%$ fetal calf serum, $100 \mathrm{U} / \mathrm{ml}$ of penicillin, $100 \mu \mathrm{g} / \mathrm{ml}$ of streptomycin, and $0.25 \mu \mathrm{g} / \mathrm{ml}$ of amphotericin B at $37^{\circ} \mathrm{C}$.

\subsection{Cloning splice variants of mouse EDA-A isoforms}

Total RNA was isolated from mouse keratinocytes using RNeasy Mini Kit (Qiagen, Valencia, CA) and treated with DNase I (RNase-Free DNase Set, Qiagen) and was used as a template to isolate variant forms of EDA. All primers used for cloning and expression analysis are listed in Table 1, designed from the mouse EDA-A1 cDNA sequence (GenBank accession no. AF016628) and mouse genomic sequence (genome.ucsc. edu, mouse assembly, October 2003 release). The plasmid pTRE-tight vector (BD Biosciences Clontech, Palo Alto, CA) containing a full-length mouse EDA-A1 cDNA (Fig. 1A) was constructed by synthesizing fragment 1 (mouse EDA-A1 cDNA, GenBank accession no. AF016628, nt 1-nt 330) and fragment 3 (nt 2001-nt 4956) by RT-PCR, using SuperScript One-Step RT-PCR for Long Templates (Invitrogen), with primer pairs $1 / 2$ and $5 / 6$, respectively. Cycling conditions were as follows: 1 cycle of $50{ }^{\circ} \mathrm{C} 30 \mathrm{~min}, 1$ cycle of $94{ }^{\circ} \mathrm{C} 2 \mathrm{~min}$, followed by 40 cycles of $94^{\circ} \mathrm{C} 30 \mathrm{~s}, 50{ }^{\circ} \mathrm{C} 60 \mathrm{~s}, 68^{\circ} \mathrm{C} 6 \mathrm{~min}$, and a final cycle of $72{ }^{\circ} \mathrm{C} 10 \mathrm{~min}$. Fragment 1 and fragment 3 PCR products were digested with NheI and NgoMIV, and SphI and NotI, respectively. Fragment 2 (mouse EDA-A1 nt 295-nt 2021) was isolated by digestion of pCI-neo-EDA-A1 (Srivastava et al., 2001) with NgoMIV and SphI. All fragments were separated on low-melt agarose gel and purified using QIAquick gel extraction Kit (Qiagen). Fragment 1, fragment 2, and fragment 3 were simultaneously ligated into the pTRE-tight vector (BD Biosciences Clontech) digested with NheI and NotI. The construct was verified by sequencing compared to the mouse EDA-A1 cDNA sequence and mouse genomic sequence (see Section 3.1).

Table 1

Primer sequences

\begin{tabular}{|c|c|c|c|}
\hline No. & Location & Direction & Sequence $\left(5^{\prime}-3^{\prime}\right)$ \\
\hline \multicolumn{4}{|c|}{ (a) Mouse EDA-A isoforms } \\
\hline 1 & Exon 1 & Forward & TATAGCTAGCTGGAGCTGCACATGCGGCTGCTC \\
\hline 2 & Exon 1 & Reverse & GCCAGCGAGAGGCCAAAGAAACCCAGG \\
\hline 3 & Exon 1 & Forward & CATGGGCTACCCAGAGGTAGAGC \\
\hline 4 & Exon 9 & Reverse & CATAGGCATGCATGTGGTCATTCCTGC \\
\hline 5 & Exon 9 & Forward & GCAGGAATGACCACATGCATGCCTATG \\
\hline 6 & Exon 9 & Reverse & TATAGCGGCCGCGGTGAGGGGAGCACTTTATTTGCAAAG \\
\hline \multicolumn{4}{|c|}{ (b) Mouse EDA isoforms including exon $8^{\prime}$} \\
\hline 7 & Exon $5-6$ & Forward & CTGGTGCTGCTGATAAAACTGGAACTC \\
\hline 8 & Exon $8^{\prime}$ & Reverse & GGACAGGAGAAGGACAAAAGTGGAG \\
\hline \multicolumn{4}{|c|}{ (c) Human EDA isoforms including exon $8^{\prime}$} \\
\hline 9 & Exon $5-6$ & Forward & GGTGCTGCTGATAAAGCTGGAACTCGAG \\
\hline 10 & Exon $8^{\prime}$ & Reverse & GCCACTCCCATGGAGGCAAACAATG \\
\hline 11 & Exon $8^{\prime}$ & Forward & CCAAGTCCACCAGTGTCCATCTACTTCC \\
\hline 12 & Exon 9 & Reverse & CACTCCACAGCAGCACTTAGAGGTTCTG \\
\hline 13 & Exon $8 \mathrm{~d}$ & Forward & CGTTGTAATGAATAGTGCTCC \\
\hline 14 & Exon 9 & Reverse & GACGCCTGCGGTATAGCAAGTGTTG \\
\hline \multicolumn{4}{|c|}{ (d) Human IgG1 Fc fragment } \\
\hline 15 & Hinge & Forward & ATATGGATCCCCCAAATCTTGTGACAAAACTCACACATGC \\
\hline 16 & $\mathrm{C}_{\mathrm{H}} 3$ & Reverse & ATATGGATCCTTTACCCGGAGACAGGGAGAGGCTCTTC \\
\hline
\end{tabular}



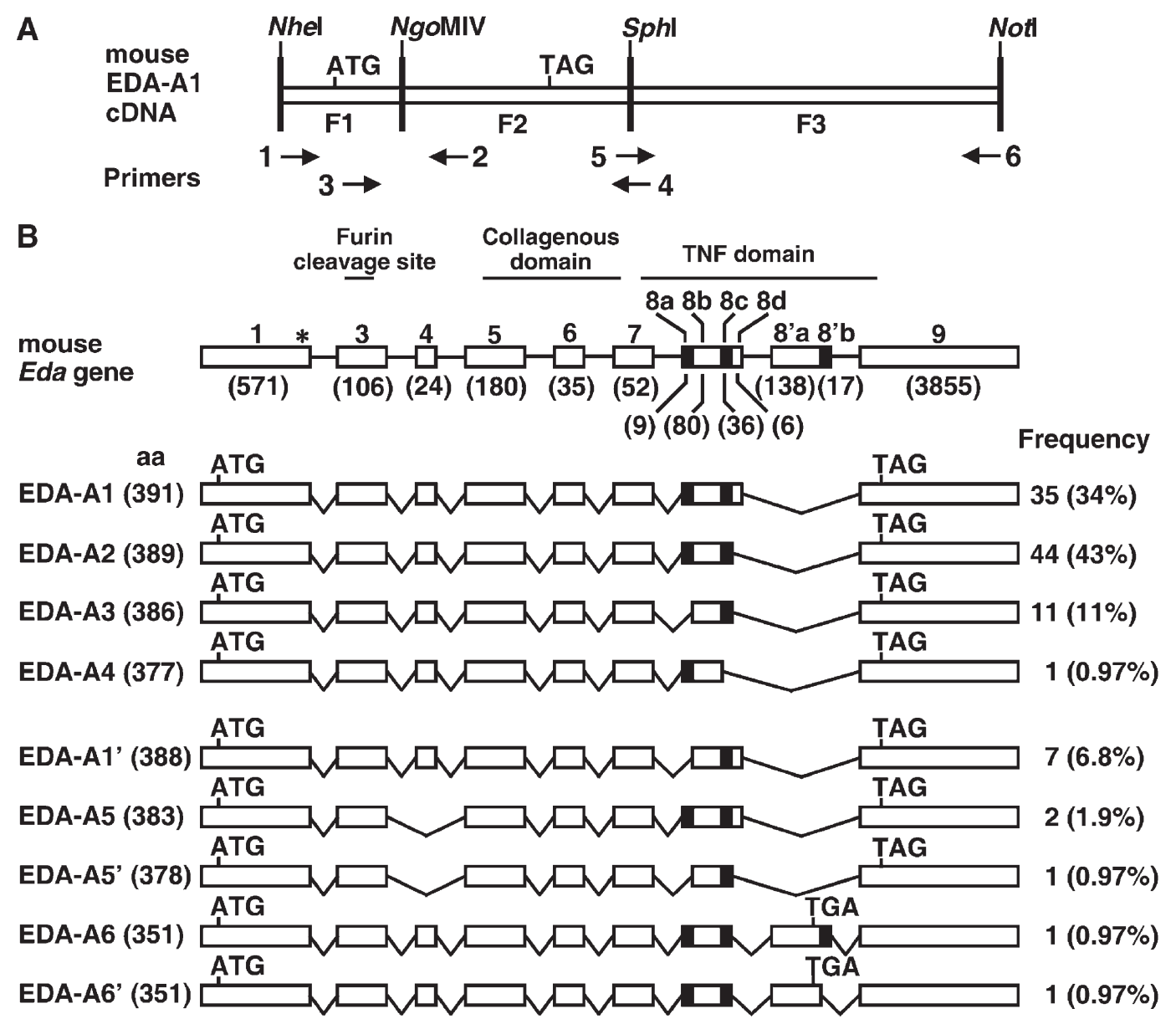

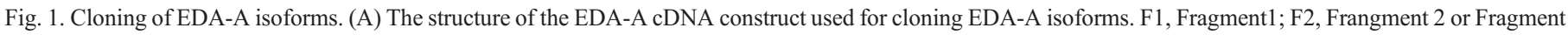

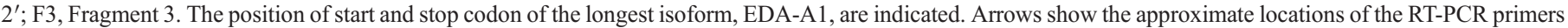

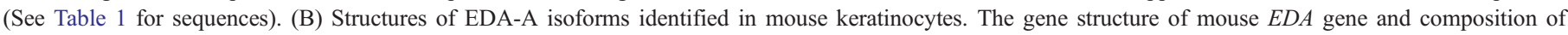

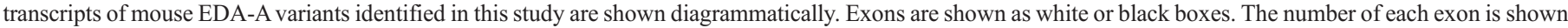

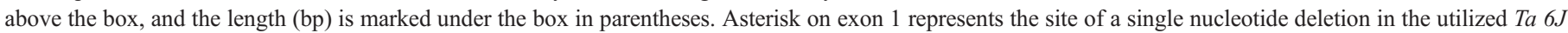

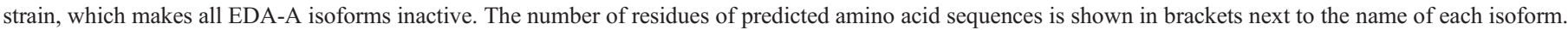

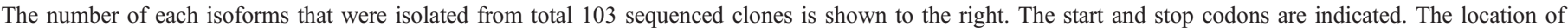
functional domains of EDA-A1 transcript is indicated on the top.

To characterize alternative open reading frames (ORF) for mouse EDA-A isoforms, fragment 2' (mouse EDA-A1 cDNA nt 175-2027) was synthesized by two-step RT-PCR with SuperScript III First-Strand Synthesis System for RT-PCR (Invitrogen) and amplified with Platinum pfx DNA Polymerase (Invitrogen). Reverse transcription was performed using an oligo-dT primer at $50{ }^{\circ} \mathrm{C}$ for $50 \mathrm{~min}$. PCR was performed (cycling conditions: $94{ }^{\circ} \mathrm{C}$ for $30 \mathrm{~s}, 53{ }^{\circ} \mathrm{C}$ for $60 \mathrm{~s}, 68{ }^{\circ} \mathrm{C}$ for 2.5 min, 40 Cycles) with primers 3 and 4 . The PCR product was digested with NgoMIV and SphI, purified as above, and ligated into the pTRE-tight-mEDA-A1 DNA digested with the same restriction enzymes and fragment 2 replaced by fragment $2^{\prime}$. The sequence of the construct was verified as above.

To examine the expression of EDA isoforms including exon $8^{\prime}$ (Fig. 1B) in vivo, total RNA was prepared from skin preparations of normal adult $\mathrm{C} 57 \mathrm{BL} / 6 \mathrm{~J}$ mouse and adult Tabby mouse $\left(\mathrm{C} 57 \mathrm{BL} / 6 \mathrm{~J}-A^{w J}-E d a^{T a-6 J} / \mathrm{J}\right.$ strain, Jackson Laboratory, Bar Harbor, ME) using TRIzol Reagent (Invitrogen). RT-PCR was done with SuperScript One-Step RT-PCR for Long Templates (Invitrogen) and primer pairs 7/8 (Table 1). Cycling conditions were 1 cycle of $50^{\circ} \mathrm{C} 30 \mathrm{~min}, 1$ cycle of $94^{\circ} \mathrm{C} 2 \mathrm{~min}$, 40 cycle of $94{ }^{\circ} \mathrm{C} 30 \mathrm{~s}, 55^{\circ} \mathrm{C} 30 \mathrm{~s}, 68^{\circ} \mathrm{C} 60 \mathrm{~s}$, and a final cycle of $72{ }^{\circ} \mathrm{C} 5 \mathrm{~min}$. RT-PCR products were analyzed on agarose gel, cloned into the pCR4-TOPO vector (Invitrogen), and verified for sequence content.

\subsection{RT-PCR analysis for EDA expression in human skin}

To characterize EDA isoforms including exon $8^{\prime}$ in human skin, total RNA from adult human skin (Stratagene, La Jolla, CA) was used as a template for RT-PCR with primer pairs 9/ 10, 11/12, and 13/14 (See Table 1 and Fig. 4) for 1 cycle of $50{ }^{\circ} \mathrm{C} 30 \mathrm{~min}, 1 \mathrm{cycle}$ of $94{ }^{\circ} \mathrm{C} 2 \mathrm{~min}, 40$ cycle of $94{ }^{\circ} \mathrm{C} 30 \mathrm{~s}$, $55^{\circ} \mathrm{C} 30 \mathrm{~s}, 68^{\circ} \mathrm{C} 60 \mathrm{~s}$, and a final cycle of $72{ }^{\circ} \mathrm{C} 5 \mathrm{~min}$. Primer sequences were based on the human EDA-A1 cDNA sequence (GenBank accession no. AF040628) and human genome sequence (genome.ucsc.edu, human assembly, July 2003). RT- 
PCR products were separated on an agarose gel, purified, and cloned into the pCR4-TOPO vector (Invitrogen), and sequenced.

\subsection{Constructions of expression vectors}

The DNA for the soluble portion of mouse EDA isoforms was PCR amplified for different isoforms as indicated in parenthesis; A1 (for aa 160-391), A2 (aa 160-389), A1' (aa 160-388), A5 (aa 160-383), and A5' (aa 160-378) from plasmids containing full-length cDNA of corresponding isoforms. The amplified products were inserted into a pFLAG-CMV1 vector (SigmaAldrich, St. Louis, MO) to express the soluble protein with an Nterminal FLAG tag. These protein fragments are referred to in the text as FLAG-EDA-A1, FLAG-EDA-A2, FLAG-EDA-A1', FLAG-EDA-A5, and FLAG-EDA-A5'. Full-length cDNAs containing mouse EDAR, XEDAR, and TROY were cloned by RT-PCR using SuperScript One-Step RT-PCR for Long Templates (Invitrogen) using total RNA from mouse skin keratinocytes as template. Expression vectors encoding fulllength EDAR, XEDAR, and TROY without a tag (flEDAR, flXEDAR, and flTROY) were constructed by inserting their cDNAs (including a stop codon) into pFLAG-CMV5.1a vector (Sigma-Aldrich). cDNAs corresponding to the extracellular portion of the mouse EDAR (aa 21-189), XEDAR (aa 2-137), and TROY (aa 30-168) were amplified from their respective full-length cDNAs by PCR, and inserted into the HindIII-BamHI site of the modified pFLAG-CMV1 vector by deleting the FLAG tag and adding a hemagglutinin (HA) tag into the SmaI site to express an extracellular part of a receptor with a C-terminal HA tag (EDAR-HA, XEDAR-HA, and TROY-HA). An expression vector for the extracellular part of XEDAR fused to the hinge and $\mathrm{Fc}$ portion of human IgG1 and an $\mathrm{HA}$ tag at the $\mathrm{C}$-terminus (XEDAR-Fc-HA) was constructed by inserting a cDNA of human IgG1 (GenBank accession no. AAC82527; aa 99-329) into the BamHI site of XEDAR-HA. cDNA of human IgG1 was obtained by RT-PCR using SuperScript One-Step RT-PCR for Long Templates (Invitrogen) from total RNA of human spleen (BD Biosciences Clontech, Palo Alto, CA) with primer 15 and 16 (Table 1).

\subsection{Luciferase reporter assays}

$293 \mathrm{~T}$ cells were transfected in subconfluent cultures in a $60 \mathrm{~mm}$ dish with $1 \mu \mathrm{g}$ of each test plasmid using liposomes (SuperFect; Qiagen, Valencia, CA). An expression vector containing a FLAG-tagged soluble EDA (FLAG-EDA-A1, FLAG-EDA-A2, FLAG-EDA-A1' ${ }^{\prime}$, FLAG-EDA-A5, or FLAGEDA-A $5^{\prime}$ ) was transfected to test NF-kB activation activity of EDA isoforms, and an expression vector encoding a full-length receptor (flEDAR, flXEDAR, or flTROY) was transfected with or without FLAG-EDA-A5 ${ }^{\prime}$ to analyze receptor specificity of EDA-A5'. An NF-kB-driven firefly luciferase reporter plasmid (PathDetect NF-кB cis-Reporting System; Stratagene; $1 \mu \mathrm{g} /$ dish) and a control plasmid encoding Renilla luciferase $(1 \mu \mathrm{g} /$ dish) (pGL4.74 [hRluc/TK] vector; Promega, Madison, WI; $0.1 \mu \mathrm{g} /$ dish) were co-transfected in each experiment. Cells were harvested $36 \mathrm{~h}$ after transfection and extracts were assayed for firefly luciferase activity and Renilla luciferase activity using a Dual-Luciferase Reporter Assay System (Promega) according to the manufacturer's protocol. Transfection efficiency was normalized to Renilla luciferase activity.

\subsection{Immunoprecipitation and Western blot}

293T cells were transfected with expression vectors using Superfect (Qiagen) to synthesize target proteins that were secreted into culture medium. Twelve hours after transfection the culture medium was changed to serum-free medium (293 SFM II; Invitrogen), and then harvested $36 \mathrm{~h}$ after the medium change. Protease inhibitors $(5 \mu \mathrm{l} / \mathrm{ml}$; PROTEASE INHIBITOR COCKTAIL, P 8340, Sigma-Aldrich) were added to the collected medium, and it was stored at $4{ }^{\circ} \mathrm{C}$ until use for immunoprecipitation assays. To test for binding of EDA isoforms with receptors, an expression vector encoding the soluble form of EDA with a FLAG tag (FLAG-EDA-A1, FLAG-EDA-A2, FLAG-EDA-A1', FLAG-EDA-A5, or FLAGEDA-A5') or the extracellular part of receptor with an HA tag (EDAR-HA, XEDAR-Fc-HA, or TROY-HA) were transfected separately $(2 \mu \mathrm{g} / 60 \mathrm{~mm}$ dish). EDA protein and receptor protein were interacted in various combinations by gentle mixing of the collected medium at $4{ }^{\circ} \mathrm{C}$ overnight, the sample was then concentrated by ultrafiltration (Amicon Ultra; MILLIPORE, Cork, Ireland) and used for immunoprecipitation with an antiHA antibody according to manufacturer's protocol (Anti-HA Immunoprecipitation Kit; Sigma-Aldrich). Immunoprecipitated proteins were eluted by boiling in sample buffer, separated by SDS-polyacrylamide gel electrophoresis, transferred to a nitrocellulose membrane, and detected by Western blot with an anti-HA antibody (HA-probe (Y-11); Santa Cruz Biotechnology, Santa Cruz, CA) or an anti-FLAG antibody (ANTI-FLAG M2 Monoclonal Antibody, Sigma-Aldrich). Immunoreactive proteins were visualized by enhanced chemiluminescence (Western Blotting Luminol Reagent, Santa Cruz). To test for binding of EDA-A5' with TROY, $1 \mu \mathrm{g}$ of each expression vector encoding the soluble form of EDA-A5' with a FLAG tag (FLAG-EDAA5') and the extracellular part of TROY receptor with an HA tag (TROY-HA) were co-transfected, and the medium was used for immunoprecipitation. The protocol above was modified to use agarose beads conjugated with an anti-FLAG antibody (ANTIFLAG M2-Agarose Affinity Gel, Sigma-Aldrich) instead of an anti-HA antibody in a buffer containing $20 \mathrm{mM}$ Tris (pH 7.4), $150 \mathrm{mM} \mathrm{NaCl}$, and $0.05 \%$ Triton-X.

\section{Results}

\subsection{Cloning of EDA-A isoforms from C57BL/6 mouse keratinocytes}

Isolation of many EDA isoforms is difficult from adult skin, where expression is very low (Kere et al., 1996); and even in embryonic skin, high expression is seen only in hair bulb cells (Mikkola et al., 1999). We therefore used mouse keratinocytes expressing EDA at a very high level to clone out rare EDA isoforms. A cDNA pool was synthesized from C57BL/6 
keratinocytes using primers 3 and 4 (Table 1), and 103 clones resulting from cloning of RT-PCR products of the F2' fragment (spanning the entire coding region of the longest isoform EDAA1; Fig. 1A) were sequenced. Nine different transcript isoforms were inferred. They included the four EDA-A isoforms previously reported in mice (EDA-A1, A2, A3, and A4) and five novel isoforms, designated along with their corresponding GenBank accession numbers, as EDA-A1' (DQ168877); EDAA5 (DQ168878); EDA-A5' (DQ168879); EDA-A6 (DQ168880) and EDA-A6' (DQ168881) (Fig. 1B).

The sequence of the EDA-A1 isoform from the isolated clones (DQ168876) differs from the previously deposited mEDA-A1 cDNA sequence (AF016628) by 26 single nucleotide changes, 25 of them in non-coding sequence and one in the coding region resulting in a silent mutation. Among the 26 single nucleotide changes, 7 are single nucleotide deletions and 5 are single nucleotide insertions. However, the sequence determined here matches perfectly with the independently assembled genomic sequence, so that mismatches in the previously published cDNA sequence are probably errors rather than representing polymorphism. It may be relevant that the products reported here were all generated with high fidelity polymerases, whereas the earlier sequences were derived using the more error-prone Taq polymerase.
The EDA-A1' isoform differs from EDA-A1 by alternate splicing of exon 8 , using an alternative splice acceptor site nine base pairs downstream of that used for EDA-A1. This change maintains the ORF in frame and terminates at the normal TAG termination codon, but leads to a peptide 3 amino acids shorter (388 instead of 391). Based on the yield of isoforms, EDA-A1' accounts for $\sim 7 \%$ of EDA-A transcripts.

EDA-A5 and EDA-A5', each recovered at the $1 \%$ level, represent a new class of transcript that completely skips exon 4 . The two differ from each other in their exon 8 structures. EDAA5 resembles EDA-A1 in its exon 8 structure; in contrast, A5' resembles EDA-A3 in using the alternative splice acceptor site 9 bp downstream normally found in EDA-A1 form, but also uses an alternative splice donor site at its $3^{\prime}$ end, 6 bases short of that found in EDA-A1. Once again, these changes remove an integral number of coding triplets, thus maintaining the open reading frame. The putative protein product of EDA-A5 is 383 amino acids long; that of EDA-A5', 378.

EDA-A6 and A6', each at about the 1\% level, are similar in structure to EDA-A2 up to exon 8, but then diverge by acquiring a new $156 \mathrm{bp}$ exonic sequence (Fig. 2A) from within sequence that is in intron 8 in the EDA-A1 isoform. We designate the new exon as exon $8^{\prime}$ (Fig. 1B) to maintain correspondence of structure with the originally defined isoforms. EDA-A6 $6^{\prime}$ is

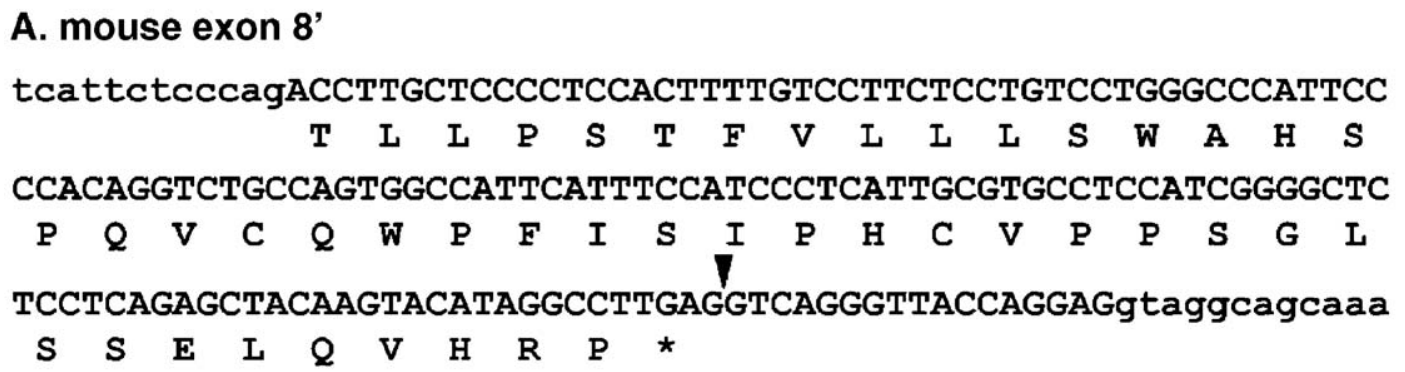

\section{B. human exon 8'}

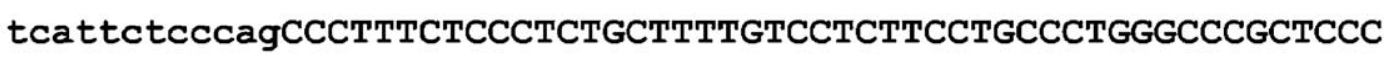
$\begin{array}{llllllllllllllll}P & F & \text { L } & \text { P } & \text { L } & \text { L } & \text { L } & \text { S } & \text { S } & \text { S } & \text { C } & \text { P } & \text { G } & \text { P } & \text { A } & \text { P }\end{array}$ CAAGTCCACCAGTGTCCATCTACTTCCGTTCCCCCATTGTTTGCCTCCATGGGAGTGGCT $\begin{array}{lllllllllllllllllllll}Q & V & \text { H } & \text { Q } & \text { C } & \text { P } & \text { S } & \text { T } & \text { S } & \text { V } & \text { P } & \text { P } & \text { L } & \text { F } & \text { A } & \text { S } & M & G & \text { V } & \text { A }\end{array}$ CCTTGGAGATAAAGGAAGATGAGCCATGAGGTTGGGATTTTTGTGGGATGGGCAGCAAGC $P$ W $R$ *

AGGCAGGTGGACAGAAGGACTATGGAGCCAACAGATGGGTGTCACATGAGAGCGGCCAGA

GAAGgtgggctaacaagcatctgctaggcacactgagcaccccgctgtagcttgggtcct

\section{C. alignment}

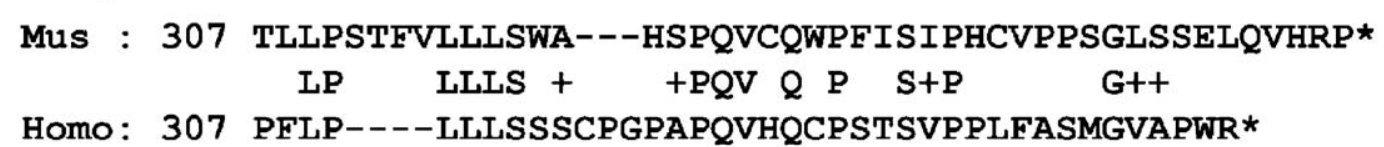

Fig. 2. The cDNA and predicted amino acid sequences of a unique exon in new EDA isoforms. (A) Mouse exon $8^{\prime}$. The boundary between exon $8^{\prime} \mathrm{a}$ and exon $8^{\prime} \mathrm{b}$ is shown by an arrowhead. (B) Human exon $8^{\prime}$. The flanking intronic nucleotide sequences are shown by lower-case letters. (C) The alignment of the predicted amino acid sequences of exon $8^{\prime}$ in mouse and human EDA. Fully conserved residues are shown between the sequences, and partially conserved residues are indicated by plus signs. 


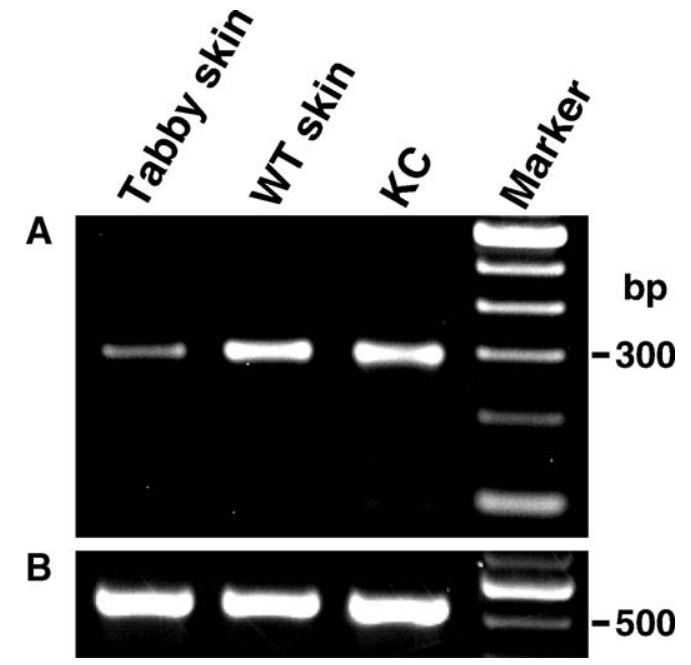

Fig. 3. Expression of new EDA isoforms in mouse skin. (A) EDA isoforms encompassing exon $8^{\prime}$. RT-PCR was performed using total RNA extracted from cultured mouse keratinocyte cells, adult wild-type mouse skin, and adult Tabby mouse skin with primers within exon 5,6 , and $8^{\prime}$. Calculated amplicon size is 291 bp. (B) $\beta$-actin. RT-PCR was performed using the same total RNAs with primers for mouse $\beta$-actin. Amplicon size is $514 \mathrm{bp}$. Marker, molecular marker (100 bp DNA Ladder, Invitrogen); KC, cultured mouse keratinocyte cells; WT skin, adult wild-type mouse skin; Tabby skin, adult Tabby mouse skin.

generated by the use of an alternative splice donor site 17 base pairs $5^{\prime}$ of that used in EDA-A6, in exon $8^{\prime}$ (Fig. 1B, 2A). The addition of exon $8^{\prime}$ notably introduces an in-frame stop codon TGA, resulting in a predicted protein of 351 amino acids for both A6 and A6' forms.

Overall, EDA-A1 and EDA-A2 remain the dominant isoforms, accounting for $34 \%$ and $43 \%$ of the total. As a first approximation based on the frequency of isoforms the newly defined isoforms account for $12 \%$ of the total, with EDA-A1' representing $7 \%$.
3.2. Expression of the most divergent new isoforms in mouse and human skin

To determine whether the new isoforms $\mathrm{A} 6$ and $\mathrm{A} 6^{\prime}$, which include the new different exon $8^{\prime}$, are expressed in mouse skin as well as in cultured keratinocytes, RT-PCR analysis was performed using primers 7 and 8 (Table 1). Expression was detected in back skin of both adult wild-type mouse and Tabby mouse (Fig. 3). The identity of RT-PCR products was confirmed by sequencing of 14 clones, all of which had the exon 8 -exon $8^{\prime}$ boundaries of EDA-A6 and A6 $6^{\prime}$. The isoforms containing exon $8^{\prime}$ are truly expressed in vivo.

The presence of human homologues of EDA-A6 and A6' (with exon $8^{\prime}$ ) was also confirmed by RT-PCR using primer pairs 9/10 and 11/12 (Table 1, Fig. 4A) on cDNA derived from human skin RNA. These primer sequences were designed based on the human EDA-A1 cDNA sequence (AF040628) and human genome sequence (genome.ucsc.edu, human assembly, July, 2003). The presence of the human homologue of exon $8^{\prime}$ (Fig. 2B) was confirmed by sequencing 19 clones derived from primers $9 / 10$ and 11 clones from primers $11 / 12$. The cDNA sequence aligned perfectly with human genome sequence, and the splice sites followed the GT-AG rule. Also, the sequence included an in-frame stop codon, as in mouse isoforms. Unlike the corresponding mouse isoforms, no alternative splicing events were detected in exon $8^{\prime}$ sequence, but the use of 3 different $3^{\prime}$ splice donor sites in exon 8 (Fig. 4B) was detected. The majority (17 of 19 clones) used the same site as EDA-A2, as also used in the mouse isoforms EDA-A6 and A6 ${ }^{\prime}$. The other two clones had splicing typical of EDA-A1 or EDA-A4 isoforms. All these isoforms terminated at the same TAA in exon $8^{\prime}$. The isoforms, including the novel exon, are thus expressed in both human and mouse. Comparisons of the

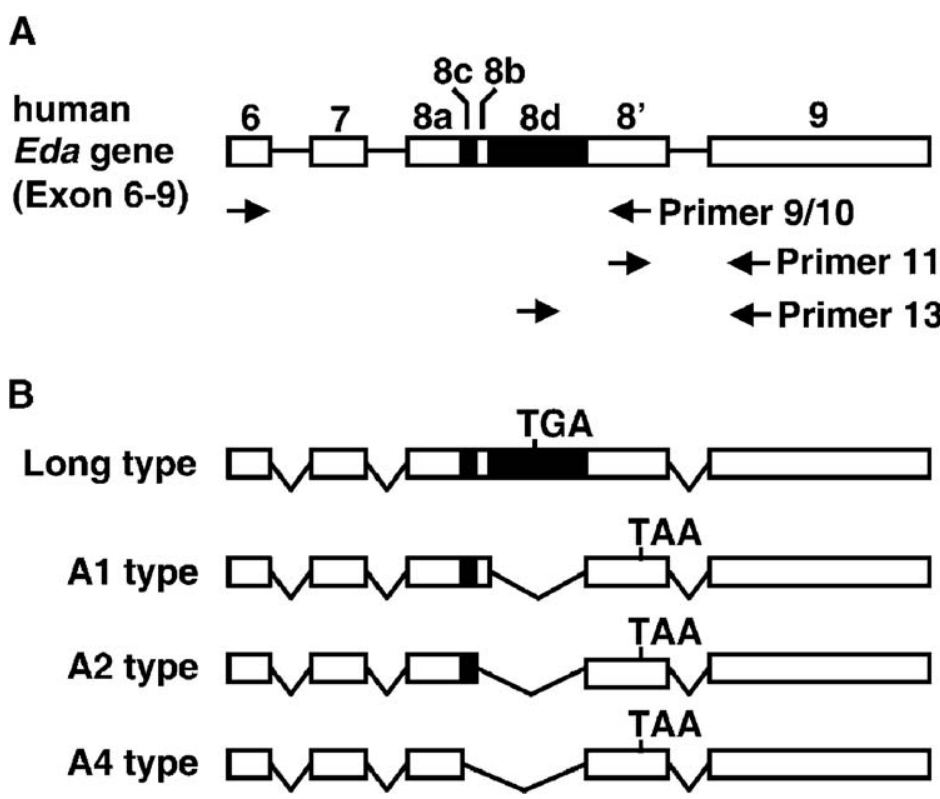

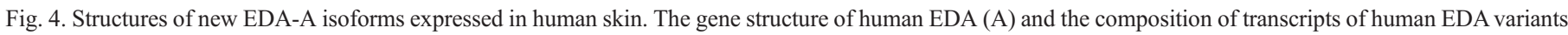

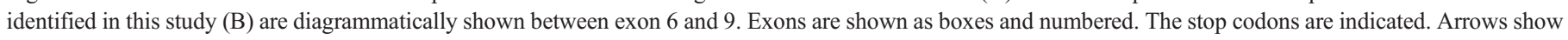
the approximate locations of the RT-PCR primers used to detect the transcripts (See Table 1 for sequences). 


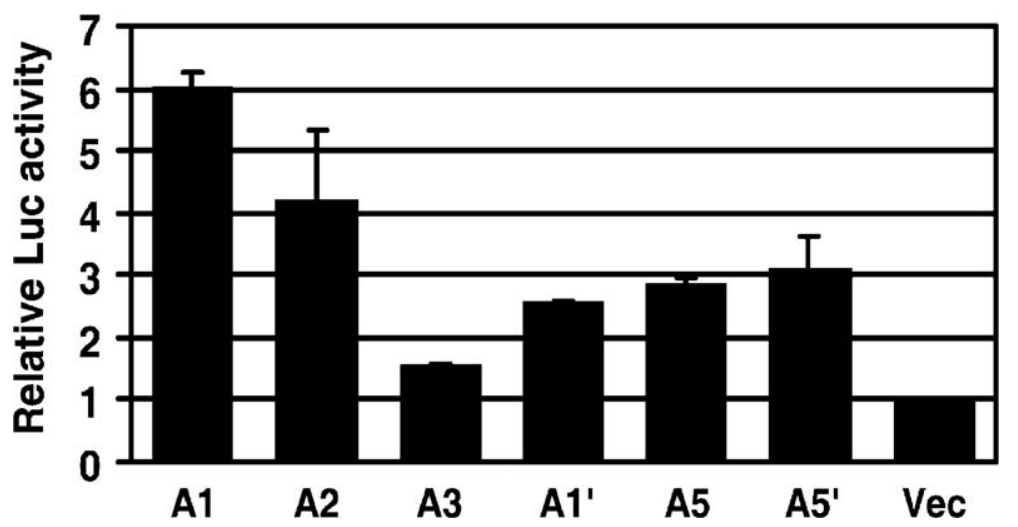

Fig. 5. Activation of NF- $\kappa$ B by EDA isoforms. $293 \mathrm{~T}$ cells were transiently transfected with an EDA isoform-encoding plasmid or an empty vector along with a NF- $\kappa \mathrm{B}$ driven luciferase (Luc) reporter vector. Cells were lysed $36 \mathrm{~h}$ after transfection and extracts were used for luciferase assay. The values shown are averages (means $\pm \mathrm{SD}$ ) of three independent experiments.

inferred amino acid sequences of exon $8^{\prime}$ in mouse and human isoforms are shown in Fig. 2C.

The repertoire of EDA isoforms is likely to be even larger than listed in Fig. 1B. For example, we recovered a very minor longer PCR product from human skin total RNA with primer pair $9 / 10$ in one experiment. Sequencing showed that it included all of the intronic sequence between exon $8 \mathrm{~b}$ and exon $8^{\prime}$. This isoform has an in-frame stop codon, TGA (Fig. 4B). Sequencing the RT-PCR products using primer 13 and 14 (Table 1, Fig. 4A) confirmed the existence of an intron between exon $8^{\prime}$ and exon 9 , indicating that this transcript is also a mature mRNA. A mouse homologue of this transcript was then also found by RT-PCR using primer pair 7/8 (Table 1) and limiting amounts of RNA templates. Again it included the whole intronic sequence between exon 8 and exon $8^{\prime}$. This additional transcript was not further studied. Skin samples expressed EDA at too low a level to permit verification of expression of other new isoforms in vivo.

\section{3. $N F-\kappa B$ activation activity and receptor specificity of new EDA isoforms}

EDA-A1 and A2 activate the NF- $\kappa$ B pathway by binding to their specific receptors, EDAR and XEDAR (Yan et al., 2000;

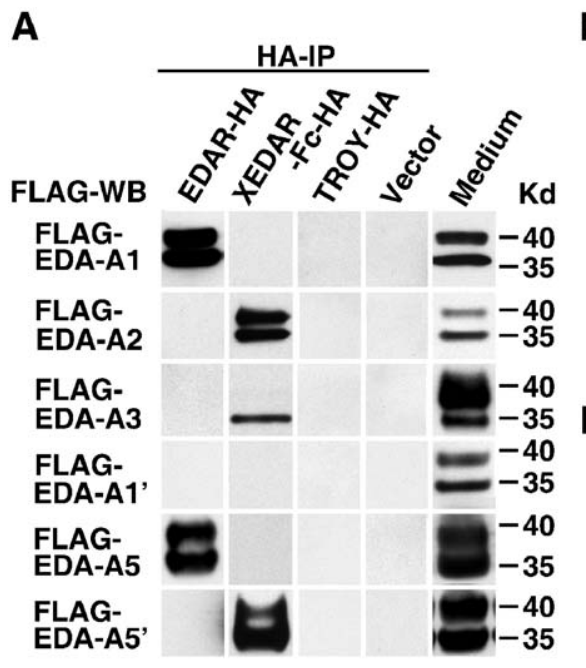

B
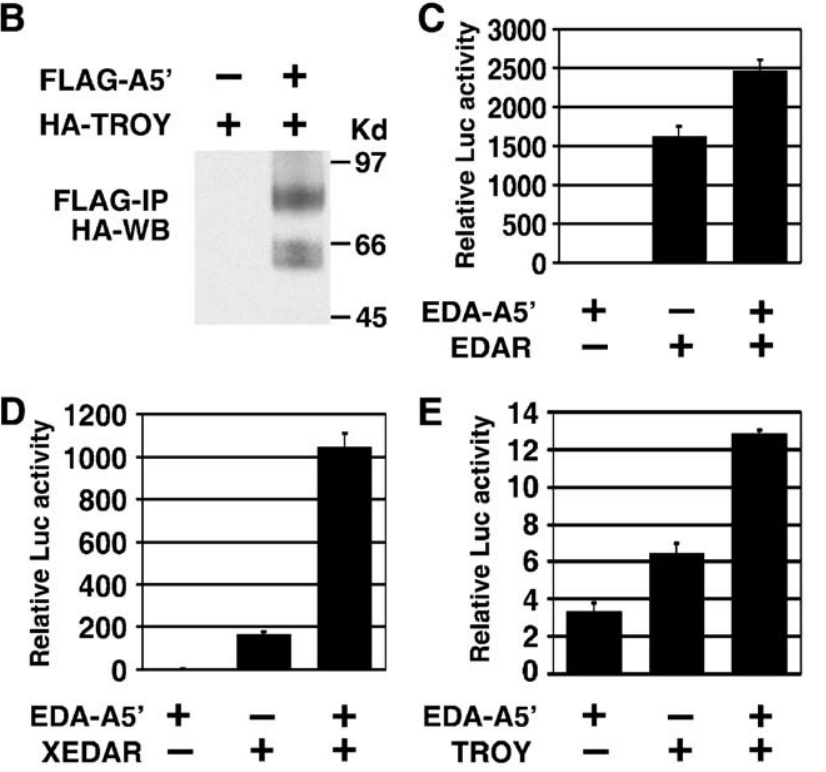

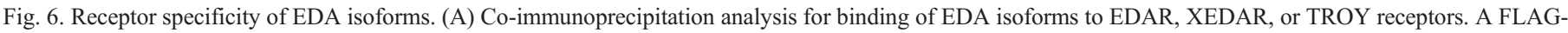

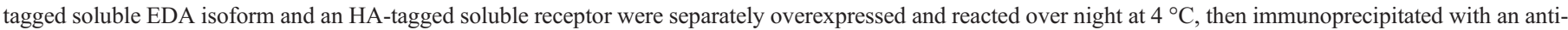

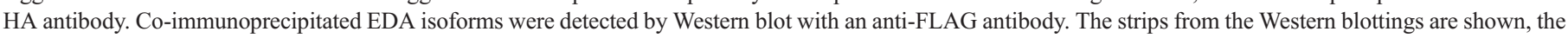

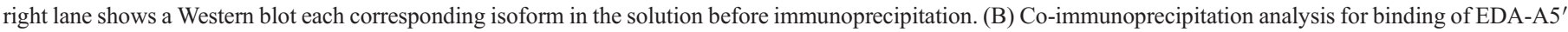

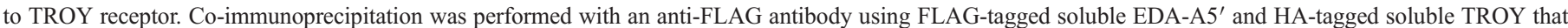

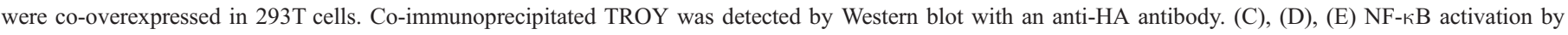

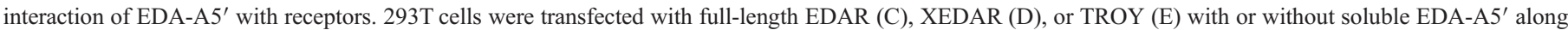

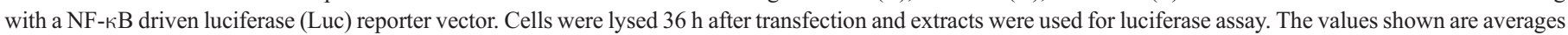
(means $\pm \mathrm{SD}$ ) of two independent experiments. 
Kumar et al., 2001; Elomaa et al., 2001; Gaide and Schneider, 2003; Chang and Chaudhary, 2004). We hypothesized that other EDA isoforms could also bind to one or the other receptor to activate NF- $\kappa$ B pathway. Three new isoforms, EDA-A1', A5, and $\mathrm{A} 5^{\prime}$, which contain a TNF motif similar to EDA-A1 or A2, were tested for their NF- $\kappa \mathrm{B}$ activation activity by transiently transfecting an expression vector encoding the respective EDA isoforms along with a NF- $\mathrm{B}$-driven luciferase reporter vector into $293 \mathrm{~T}$ cells (Fig. 5). The overexpressed EDA proteins contain a complete soluble portion of EDA that is biologically secreted by cleavage at $\operatorname{Arg}^{159}$ by furin (Elomaa et al., 2001; Schneider et al., 2001; Chen et al., 2001). The 293 cells do not express EDA, and express receptors weakly but sufficient to show activation. Transfection of all tested new isoforms induced greater than 2-fold luciferase activity compared to a vector control.

We next tested binding specificity for candidate receptors. In addition to EDAR and XEDAR, we examined TROY, which is another TNF superfamily receptor similar to EDAR and XEDAR in its structure and localization in skin (Hu et al., 1999; Kojima et al., 2000; Eby et al., 2000; Yan et al., 2000). TROY showed no binding to EDA-A1 or A2 (Kumar et al., 2001; Gaide and Schneider, 2003) isoforms, but remains a potential receptor for other EDA isoforms. A FLAG-tagged soluble EDA isoform and an extracellular part of a receptor with an HA tag were overexpressed in 293 T cells separately and used for co-immunoprecipitation assay with an anti-HA antibody followed by Western blotting with an anti-FLAG antibody (Fig. 6A). Overexpressed EDA isoforms were usually detected as double bands with slower moving species probably due to $\mathrm{N}$ linked glycosylation as determined by previous studies (Mikkola et al., 1999; Schneider et al., 2001; Chen et al., 2001; Chang and Chaudhary, 2004). The extracellular forms of EDAR, XEDAR, and TROY migrated as expected, at about 40 $\mathrm{kd}, 55 \mathrm{kd}$, and $30 \mathrm{kd}$, respectively, and dimers were detected for XEDAR and TROY. Bindings of EDA-A1/EDAR and EDAA2/XEDAR were confirmed as previously reported. EDA-A3 and $\mathrm{A} 5^{\prime}$ were co-immunoprecipitated with XEDAR, and EDAA5 with EDAR. The signal from EDA-A3 was weak and its higher molecular weight form was faint, and thus far no coimmunoprecipitate with any tested receptors has been detected for EDA-A1'.

Although Western blots showed that TROY receptor was expressed at levels comparable to EDAR or XEDAR (Supplementary Fig. 1), none of the EDA isoforms tested coimmunoprecipitated with TROY, suggesting that TROY is not a preferred receptor for any isoforms tested. However, an alternate method indicated that $\mathrm{A} 5^{\prime}$ may have low affinity to TROY. We tested interaction of EDA isoforms with TROY by "reverse IP", that is, by co-immunoprecipitation with an antiFLAG antibody using a FLAG-tagged soluble EDA isoform and an HA-tagged extracellular part of TROY receptor that were overexpressed together in 293T cells. Western blot analysis with an anti-HA antibody indicated that with this protocol a small amount of added TROY was co-immunoprecipitated with EDA-A5' (Fig. 6B); Western blot analysis (not shown) showed that less than $5 \%$ of TROY was immunopre- cipitated. To test further whether EDA-A5' is a functional ligand for XEDAR and TROY receptors, we assessed NF-кB activation by a full-length receptor in transfections with or without the soluble EDA-A5' in 293T cells using luciferase reporter assay (Fig. $6 \mathrm{C}-\mathrm{E}$ ). NF- $\kappa \mathrm{B}$ activity was increased in the presence of EDA-A5' for EDAR, XEDAR and TROY. However, a synergistic effect was clearly observed only for XEDAR, whereas the effect on TROY in the presence of EDAA5' was weak and only additive. These results indicated that EDA-A5' is a functional ligand preferentially for XEDAR; any interaction with TROY may require additional factor to be stable (see Section 4).

\section{Discussion}

The 5 novel isoforms detected are present in keratinocytes at significant though minor levels, adding up to about $12 \%$ of total transcripts. We demonstrate that at least three of them, EDAA1', EDA-A5, and EDA-A5', are functional isoforms with activation activity for the downstream transcription factor NF$\kappa \mathrm{B}$, and might thus influence skin appendage development.

The divergent novel transcripts were generated by splicing out exon 4 , using alternate splice sites in exon 8 , or including a new exon $8^{\prime}$ from within the previously defined intron 8 region. Expression of the relatively frequent exon $8^{\prime}$ was confirmed in skin. No changes in exons $1,3,5,6,7$, or 9 were detected. Consequently, all novel EDA isoforms use the same unique ATG start site in exon 1 (contained in primer 3 used to amplify all isoforms), and retain a furin cleavage site encoded in exon 3 and a collagen domain encoded in exon 5-7, suggesting that they could form a soluble trimerized molecule, like previously known isoforms EDA-A1 and A2 (Ezer et al., 1999; Mikkola et al., 1999; Chen et al., 2001; Elomaa et al., 2001; Schneider et al., 2001).

EDA-A5 and EDA-A5' result from exon 4 skipping. Exon 4 contains 24 nucleotides that encode a linker sequence between the furin cleavage site and the collagenous domain, and deletion of exon 4 is in frame, thus causing no change in the TNF domain. Exon 4 can be dispensable for function of a TNF superfamily ligand, because immunoprecipitation assays showed that EDA-A5 and EDA-A5' could still bind to receptors, and both activate $\mathrm{NF}-\kappa \mathrm{B}$. Consistent with these data, truncated EDA-A1 and EDA-A2, which also lacked the amino acids encoded by exon 4 still bound to their specific receptors and activated NF- $\kappa \mathrm{B}$, and truncated EDA-A1 could rescue part of the Tabby phenotype (Yan et al., 2000; Schneider et al., 2001; Koppinen et al., 2001; Gaide and Schneider, 2003). Furthermore, no mutations in exon 4 have been reported to cause human X-linked anhidrotic ectodermal dysplasia patients (Hashiguchi et al., 2003; Visinoni et al., 2003).

Changes in the exon 8 region coding for TNF motif could be more consequential. Exon 8 has two $5^{\prime}$ acceptor sites and three $3^{\prime}$ donor sites, and five different forms of exon 8 could be potentially generated by alternative splicing. One of these was found in this study in EDA-A1'. Two amino acids encoded at the $3^{\prime}$ end of exon 8 are essential to determine ligand/receptor specificity for EDA-A1/EDAR or EDA-A2/XEDAR (Yan et al., 
2000; Hymowitz et al., 2003). As expected, co-immunoprecipitation assays demonstrated that EDA-A3 and EDA-A5', which have a TNF motif similar to A2, lacking the two amino acids in exon 8, bound to XEDAR; whereas EDA-A5, which has an TNF motif identical to A1, bound to EDAR. Biological activity of EDA-A5'/XEDAR interaction was demonstrated by increased NF- $\kappa B$ activity in transfected cells. Based on their receptor specificity, EDA-A5 and EDA-A3/A5' could act similarly to EDA-A1 and A2.

In extreme cases of exon 8 changes, EDA-A4 lacked 12 amino acids at the $3^{\prime}$ end of the exon, and EDA-A6 and A6' were unique variants that encompass an additional exon (exon $8^{\prime}$ ) between exon 8 and 9. They were confirmed to be expressed in both human and mouse skin, and contained a stop codon that truncated the TNF motif. Such truncated forms would almost certainly not function as TNF ligands.

We failed to detect the binding of EDA-A1' to EDAR, even though EDA-A1' contains the critical two amino acids of A1 at the $3^{\prime}$ end of exon 8. However, EDA-A1' lacks the 3 amino acids at $5^{\prime}$ end of exon 8 , and this might decrease the binding to EDAR; nevertheless, it must bind weakly to EDAR or other receptors, because it activated NF- $\kappa B$ in vitro (see Fig. 5).

We anticipated that some of the EDA isoforms could be ligands for a TNF superfamily receptor, TROY (also known as TNFRSF19, TRADE, or TAJ), which functions in the nervous system but is similar to EDAR and XEDAR (Hu et al., 1999; Kojima et al., 2000; Eby et al., 2000; Yan et al., 2000; Park et al., 2005; Shao et al., 2005). However, it has no known ligand in skin. Co-immunoprecipitation assays suggested EDA-A5' might bind to TROY; but the results were inconclusive, because the interactions between EDA-A5' and TROY were weak, and were detected when they were overexpressed together in 293T cells, but not when they were mixed after independent expression in different cell cultures. Also, NF- $\mathrm{kB}$ activation activity of TROY receptor was very low, and was not synergistically increased by a soluble EDA-A5'ligand. Recent studies have demonstrated that TROY forms a functional receptor complex by interacting to RTN4R (also known as $\mathrm{NgR}$ ) and LRRN6A (also known as LINGO-1) in neural cells (Park et al., 2005; Shao et al., 2005), so that it might require additional components to stabilize otherwise weak interactions. A more direct biological experiment to assess the relevance of TROY to skin appendage formation should be provided by the study of a TROY-mutant mouse, which is currently being bred from mutated ES cells (work in progress).

The extensive variation in the TNF ligand portion of the molecule hints that some isoforms, even including truncated ones, might modulate the action of others. The structure of EDA (Mikkola et al., 1999; Ezer et al., 1999; Elomaa et al., 2001) creates several possibilities for modulation or competitive interactions, because all EDA-A isoforms, including the newly found ones, can potentially form trimers in various combinations, and heterotrimers might be required for the full range of EDA function. Even low levels of an isoform could have a significant effect, and some of the rare isoforms - like EDA-A1 and A2 (Yan et al., 2000) — might also be differentially highly expressed at specific developmental stages. There are hints of such possible competitive or modulatory interactions of isoforms in the reported inhibition of zigzag hair formation by an EDA-A1 transgene introduced into wild-type mice (Cui et al., 2003; Mustonen et al., 2003); and in the modification of hair in mice treated with EDA-A2 protein in conjunction with EDAA1 (Gaide and Schneider, 2003). Upstream regulators of Edar signaling, such as Wnt (Durmowicz et al., 2002; Laurikkala et al., 2002), should be comparable for all isoforms, but some isoforms could differentially affect Edar activation of downstream pathways involved in hair follicle development, like Shh (Laurikkala et al., 2002).

\section{Acknowledgements}

The authors thank Chris Ottolenghi for technical assistance, and Ramaiah Nagaraja for critical reading of the manuscript. Tsuyoshi Hashimoto is a 2004 Japan Society for the Promotion of Science (JSPS) Fellow in Biomedical and Behavioral Research at NIH. This research was supported by the Intramural Research Program of the NIH, National Institute on Aging.

\section{Appendix A. Supplementary data}

Supplementary data associated with this article can be found, in the online version, at doi:10.1016/j.gene.2005.11.003.

\section{References}

Bayes, M., et al., 1998. The anhidrotic ectodermal dysplasia gene (EDA) undergoes alternative splicing and encodes ectodysplasin-A with deletion mutations in collagenous repeats. Hum. Mol. Genet. 7, 1661-1669.

Chang, B., Chaudhary, P.M., 2004. High level production and one-step purification of biologically active ectodysplasin A1 and A2 immunoadhesins using the baculovirus/insect cell expression system. Protein Expr. Purif. $37,162-169$.

Chen, Y., et al., 2001. Mutations within a furin consensus sequence block proteolytic release of ectodysplasin-A and cause X-linked hypohidrotic ectodermal dysplasia. Proc. Natl. Acad. Sci. U. S. A. 98, 7218-7223.

Courtois, G., et al., 2003. A hypermorphic IkappaBalpha mutation is associated with autosomal dominant anhidrotic ectodermal dysplasia and $\mathrm{T}$ cell immunodeficiency. J. Clin. Invest. 112, 1108-1115.

Cui, C.Y., et al., 2003. Inducible mEDA-A1 transgene mediates sebaceous gland hyperplasia and differential formation of two types of mouse hair follicles. Hum. Mol. Genet. 12, 2931-2940.

Cui, C.Y., Smith, J.A., Schlessinger, D., Chan, C.C., 2005. X-linked anhidrotic ectodermal dysplasia disruption yields a mouse model for ocular surface disease and resultant blindness. Am. J. Pathol. 167, 89-95.

Durmowicz, M.C., Cui, C.Y., Schlessinger, D., 2002. The EDA gene is a target of, but does not regulate Wnt signaling. Gene 285, 203-211.

Eby, M.T., Jasmin, A., Kumar, A., Sharma, K., Chaudhary, P.M., 2000. TAJ, a novel member of the tumor necrosis factor receptor family, activates the cJun N-terminal kinase pathway and mediates caspase-independent cell death. J. Biol. Chem. 275, 15336-15342.

Elomaa, O., Pulkkinen, K., Hannelius, U., Mikkola, M., Saarialho-Kere, U., Kere, J., 2001. Ectodysplasin is released by proteolytic shedding and binds to the EDAR protein. Hum. Mol. Genet. 10, 953-962.

Ezer, S., Bayes, M., Elomaa, O., Schlessinger, D., Kere, J., 1999. Ectodysplasin is a collagenous trimeric type II membrane protein with a tumor necrosis factor-like domain and co-localizes with cytoskeletal structures at lateral and apical surfaces of cells. Hum. Mol. Genet. 8, 2079-2086. 
Ferguson, B.M., Brockdorff, N., Formstone, E., Ngyuen, T., Kronmiller, J.E., Zonana, J., 1997. Cloning of Tabby, the murine homolog of the human EDA gene: evidence for a membrane-associated protein with a short collagenous domain. Hum. Mol. Genet. 6, 1589-1594.

Gaide, O., Schneider, P., 2003. Permanent correction of an inherited ectodermal dysplasia with recombinant EDA. Nat. Med. 9, 614-618.

Hashiguchi, T., Yotsumoto, S., Kanzaki, T., 2003. Mutations in the ED1 gene in Japanese families with X-linked hypohidrotic ectodermal dysplasia. Exp. Dermatol. 12, 518-522.

Headon, D.J., Overbeek, P.A., 1999. Involvement of a novel Tnf receptor homologue in hair follicle induction. Nat. Genet. 22, 370-374.

Hu, S., Tamada, K., Ni, J., Vincenz, C., Chen, L., 1999. Characterization of TNFRSF19, a novel member of the tumor necrosis factor receptor superfamily. Genomics 62, 103-107.

Hymowitz, S.G., et al., 2003. The crystal structures of EDA-A1 and EDA-A2: splice variants with distinct receptor specificity. Structure (Camb) 11, $1513-1520$

Kere, J., et al., 1996. X-linked anhidrotic (hypohidrotic) ectodermal dysplasia is caused by mutation in a novel transmembrane protein. Nat. Genet. 13, 409-416.

Kojima, T., et al., 2000. TROY, a newly identified member of the tumor necrosis factor receptor superfamily, exhibits a homology with Edar and is expressed in embryonic skin and hair follicles. J. Biol. Chem. 275, 20742-20747.

Koppinen, P., Pispa, J., Laurikkala, J., Thesleff, I., Mikkola, M.L., 2001. Signaling and subcellular localization of the TNF receptor edar. Exp. Cell Res. 269, 180-192.

Kumar, A., Eby, M.T., Sinha, S., Jasmin, A., Chaudhary, P.M., 2001. The ectodermal dysplasia receptor activates the nuclear factor-kappaB, JNK, and cell death pathways and binds to ectodysplasin A. J. Biol. Chem. 276, 2668-2677.

Laurikkala, J., et al., 2002. Regulation of hair follicle development by the TNF signal ectodysplasin and its receptor Edar. Development 129, 2541-2553.

Mikkola, M.L., et al., 1999. Ecotdysplasin, a protein required for epithelial morphogenesis, is a novel TNF homologue and promotes cell-matrix adhesion. Mech. Dev. 88, 133-146.

Monreal, A.W., Ferguson, B.M., Headon, D.J., Street, S.L., Overbeek, P.A., Zonana, J., 1999. Mutations in the human homologue of mouse dl cause autosomal recessive and dominant hypohidrotic ectodermal dysplasia. Nat. Genet. 22, 366-369.

Mustonen, T., et al., 2003. Stimulation of ectodermal organ development by Ectodysplasin-A1. Dev. Biol. 259, 123-136.
Naito, A., et al., 2002. TRAF6-deficient mice display hypohidrotic ectodermal dysplasia. Proc. Natl. Acad. Sci. U. S. A. 99, 8766-8771.

Newton, K., French, D.M., Yan, M., Frantz, G.D., Dixit, V.M., 2004. Myodegeneration in EDA-A2 transgenic mice is prevented by XEDAR deficiency. Mol. Cell. Biol. 24, 1608-1613.

Ohazama, A., et al., 2004. Traf6 is essential for murine tooth cusp morphogenesis. Dev. Dyn. 229, 131-135.

Park, J.B., et al., 2005. A TNF receptor family member, TROY, is a coreceptor with Nogo receptor in mediating the inhibitory activity of myelin inhibitors. Neuron 45, 345-351.

Schneider, P., et al., 2001. Mutations leading to X-linked hypohidrotic ectodermal dysplasia affect three major functional domains in the tumor necrosis factor family member ectodysplasin-A. J. Biol. Chem. 276, 18819-18827.

Shao, Z., et al., 2005. TAJ/TROY, an orphan TNF receptor family member, binds Nogo-66 receptor 1 and regulates axonal regeneration. Neuron 45, 353-359.

Shindo, M., Chaudhary, P.M., 2004. The ectodermal dysplasia receptor repress the Lef- $1 / \beta$-catenin-dependent transcription independent of NF- $k B$ activation. Biochem. Biophys. Res. Commun. 315, 73-78.

Srivastava, A.K., et al., 1997. The Tabby phenotype is caused by mutation in a mouse homologue of the EDA gene that reveals novel mouse and human exons and encodes a protein (ectodysplasin-A) with collagenous domains. Proc. Natl. Acad. Sci. U. S. A. 94, 13069-13074.

Srivastava, A.K., et al., 2001. Ectodysplasin-A1 is sufficient to rescue both hair growth and sweat glands in Tabby mice. Hum. Mol. Genet. 10, 2973-2981.

Visinoni, A.F., de Souza, R.L., Freire-Maia, N., Gollop, T.R., Chautard-FreireMaia, E.A., 2003. X-linked hypohidrotic ectodermal dysplasia mutations in Brazilian families. Am. J. Med. Genet. 122A, 51-55.

Yan, M., et al., 2000. Two-amino acid molecular switch in an epithelial morphogen that regulates binding to two distinct receptors. Science 290, $523-527$.

Yan, M., Zhang, Z., Brady, J.R., Schilbach, S., Fairbrother, W.J., Dixit, V.M., 2002. Identification of a novel death domain-containing adaptor molecule for ectodysplasin-A receptor that is mutated in crinkled mice. Curr. Biol. 12, 409-413.

Zhang, M., Brancaccio, A., Weiner, L., Missero, C., Brissette, J.L., 2003. Ectodysplasin regulates pattern formation in the mammalian hair coat. Genesis 37, 30-37. 